\title{
Dispersion of surface plasmon polaritons on silver film with rectangular hole arrays in a square lattice
}

\author{
Ming-Wei Tsai, ${ }^{a)}$ Tzu-Hung Chuang, Hsu-Yu Chang, and Si-Chen Lee \\ Department of Electrical Engineering, Graduate Institute of Electronics Engineering, \\ National Taiwan University, Taipei, Taiwan 106, Republic of China
}

(Received 13 March 2006; accepted 5 July 2006; published online 28 August 2006)

\begin{abstract}
Extraordinary optical transmission through a two-dimensional Ag film in the far infrared region was demonstrated. The holes were rectangular and arranged in a square lattice. When either the width or the length size of the rectangular holes was close to the half of the lattice constant $a / 2$, the degenerate $( \pm 1,0) \mathrm{Ag} / \mathrm{Si}$ or $(0, \pm 1) \mathrm{Ag} / \mathrm{Si}$ modes split into two peaks. Additionally, the surface plasmon dispersion relations of the square hole array with different aspect ratios of holes were measured. As the aspect ratio increased, the surface plasmon tended to couple strongly with the local charge dipole oscillations in a direction perpendicular to the long edges. The charge dipole oscillation parallel to the long edges gradually disappeared. This is confirmed by experiments using the polarized light. The dynamic properties of the surface plasmon dispersion relations show the interaction among localized surface plasmons and its mechanism. (C) 2006 American Institute of Physics. [DOI: 10.1063/1.2338886]
\end{abstract}

Optically thick metal films with subwavelength periodic perforated hole arrays exhibit extraordinary optical transmission. ${ }^{1,2}$ As the holes become smaller than the thickness of the metal film, flat dispersions and small transmission intensities cause a transition from surface plasmon (SP)mediated behavior to waveguide-dominated behavior. ${ }^{3} \mathrm{Re}$ cent work on the conservation of SPs and light through periodic perforated hole arrays ${ }^{4}$ has elucidated the propagation of SPs. Extraordinary optical transmission in the middle and far infrared regions has been demonstrated. ${ }^{5-7}$ When the light is normally incident on metal films, localized surface plasmons (LSPs) due to the individual hole shift the wavelength and affect the transmission intensity. ${ }^{8-13}$ These properties follow from the dipole nature of the LSP modes and can be tuned by appropriately designing of the shape of the aperture. 8,9 The dependence of the transmittance through a single hole on hole shape has been theoretically presented. ${ }^{14}$ However, the surface plasmon polaritons (SPPs) formed by the interaction of light with the grating dominate the transmission spectra even when the LSP contribution is present. The shape resonance may be regarded as the effect of the coupling between LSPs and the long edges of the rectangular hole. This resonance causes the cutoff wavelength and the transmission efficiency of the LSPs to increase with the aspect ratio $(x / y) .^{10,15}$ The difference between the cutoff wavelength of the long and short edges of the rectangular hole may be responsible for the different transmission peaks. This has potential applications in optical filters and tunable and focusing light sources. ${ }^{16-18}$ This letter studies the dynamic properties of the SPP dispersion on the extraordinary transmission in the far infrared region through a periodic perforated Ag film with a two-dimensional square array of rectangular holes. Asymmetric charge distribution at the long edges and the short edges of the perforated rectangular holes is discussed to explain the relationship between LSP waves induced by electric field oscillation and surface charge distribution.

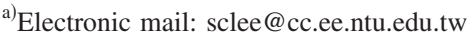

After the photoresist was spun on the silicon wafer and pattern transfer, 100-nm-thick Ag metal films were deposited and lifted off to form squared hole arrays with different rectangular holes. A Bruker IFS $66 \mathrm{v} / \mathrm{s}$ system was used to measure zero-order transmission spectra. The sample was defined as lying in the $(x, y)$ plane and was rotated around the $y$ axis in $1^{\circ}$ increments up to $\theta=50^{\circ}$, as illustrated in Fig. 1(a), allowing the dispersion relation in the $\mathbf{k}_{x}$ direction to be studied. The sample was then rotated around the $x$ axis in $1^{\circ}$ increments up to $\theta=50^{\circ}$, as shown in Fig. 1(b), allowing the dispersion relation in the $\mathbf{k}_{y}$ direction to be studied. The light is incident on the $\mathrm{Ag}$ side of the sample. The resolution of the wave number was $8 \mathrm{~cm}^{-1}$.

Figures 2(a) and 2(b) show the transmission spectra of a square array of rectangular holes at normal incidence with different aspect ratios $x / y$. The lattice constants $a$ are 10 and $9 \mu \mathrm{m}$; the aspect ratios $x / y$ of the rectangular holes vary from 1.2 to 2.2 and from 1.15 to 1.9 , as shown in Figs. 2(a) and 2(b), respectively. Figure 2(a) shows that when either the width or the length of the rectangular hole is close to half of the lattice constant $a / 2,5 \mu \mathrm{m}$, either the degenerate $( \pm 1,0)$ $\mathrm{Ag} / \mathrm{Si}$ or $(0, \pm 1) \mathrm{Ag} / \mathrm{Si}$ mode splits into two peaks at 35 and $37 \mu \mathrm{m}$. Similar results hold for a lattice constant of $9 \mu \mathrm{m}$, as sample A shown in Fig. 2(b). As the aspect ratio increases,

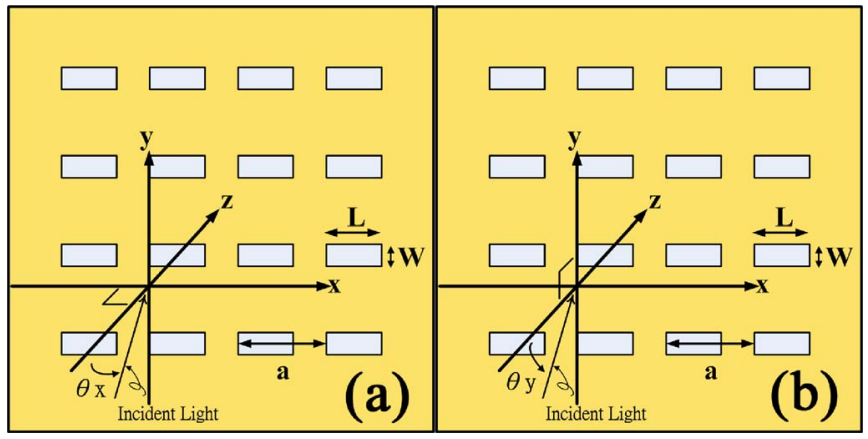

FIG. 1. (Color online) Schematic diagram showing the experiment setup. The sample is defined to lie in the $(x, y)$ plane, rotating it around (a) the $y$ axis and (b) the $x$ axis by $1^{\circ}$ increment up to $\theta=50^{\circ}$. 

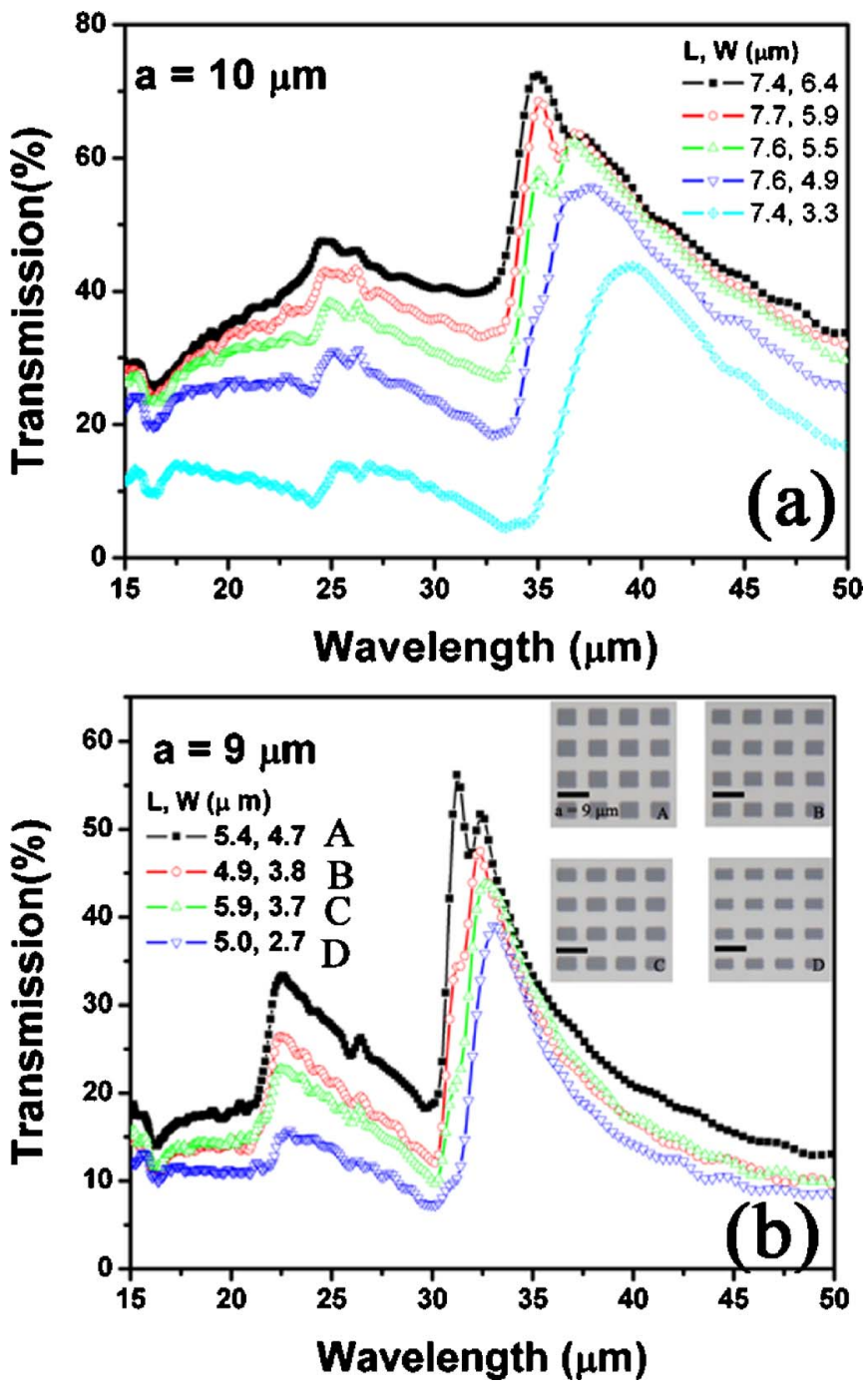

FIG. 2. (Color online) Zero-order transmission spectra of a squared hole array with different aspect ratios of the rectangular hole at normal incidence. The Ag film thickness is $100 \mathrm{~nm}$ and the lattice constants $a$ are (a) $10 \mu \mathrm{m}$ and (b) $9 \mu \mathrm{m}$. The inset of (b) shows the photographs of the samples A-D.

the wavelength of the left peak remains unchanged while that of the right peak shifts to longer wavelength [36.8-39.6 $\mu \mathrm{m}$ in Fig. 2(a)]. The transmission intensity of the left peak becomes smaller than that of the right peak. Coupling between LSPs and the long edges of the rectangular hole causes the cutoff wavelength to increase with the aspect ratio $(x / y) .^{10,15}$ The coupling efficiency of LSPs waves to the long edge of the rectangular hole exceeds that to the short edge.

Figures 3(a)-3(d) display the SPP dispersion relations of a square array of rectangular holes with a lattice constant $a$ of $9 \mu \mathrm{m}$ and the aspect ratios of $1.15(L=5.4 \mu \mathrm{m}, W$ $=4.7 \mu \mathrm{m})$ and $1.9(L=5.0 \mu \mathrm{m}, W=2.7 \mu \mathrm{m})$, respectively. The angular variation of the incident radiation in Figs. 1(a) and 1(b) is adopted to yield the results shown in Figs. 3(a) and 3(c), and 3(b) and 3(d), respectively. When the aspect ratio of the rectangular hole was 1.15 , the charge distributions at long edges and short edges of the rectangular hole are almost the same, the $(1,0) \mathrm{Ag} / \mathrm{Si}$ and $(-1,0) \mathrm{Ag} / \mathrm{Si}$ mode in Fig. 3(a) and the $(0, \pm 1) \mathrm{Ag} / \mathrm{Si}$ modes in Fig. 3(b) are all present. However, the brightness of the $(-1,0) \mathrm{Ag} / \mathrm{Si}$ mode exceeds that of the $(1,0) \mathrm{Ag} / \mathrm{Si}$ mode, because of the backward scattering of SPP waves. Finally, when the aspect ratio of the rectangular hole is 1.9 , the $(1,0) \mathrm{Ag} / \mathrm{Si}$ and Downloaded 04 Mar 2009 to 140.112.113.225. Redistribution subject

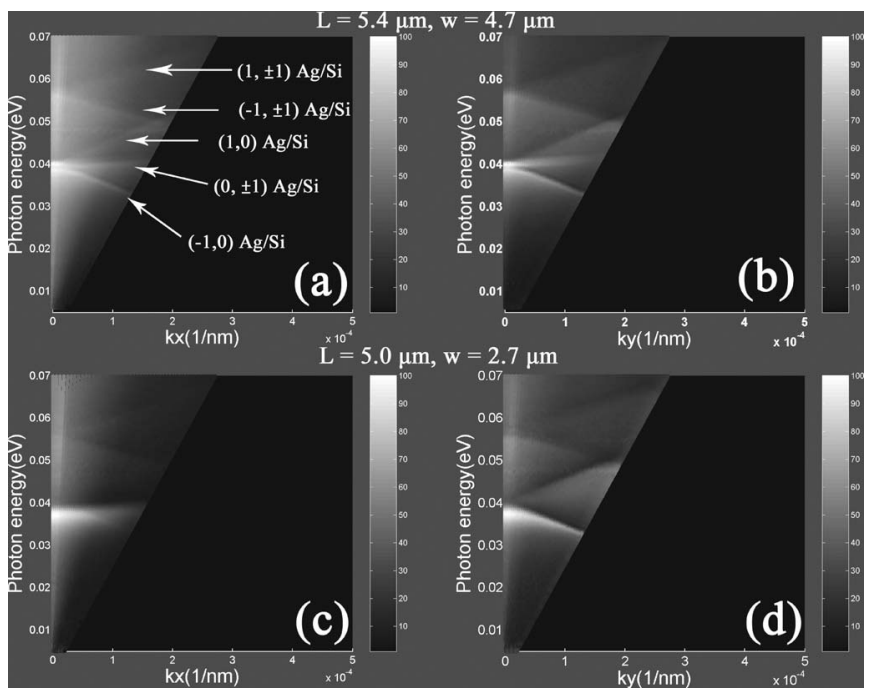

FIG. 3. Energy dispersion relation of SPPs, transmission intensity (gray scale) as a function of photon energy and $\mathbf{k}_{x}$ or $\mathbf{k}_{y}$. The length and width of the rectangular hole are $L=5.4 \mu \mathrm{m}$ and $W=4.7 \mu \mathrm{m}$ in (a) and (b), and $L$ $=5.0 \mu \mathrm{m}$ and $W=2.7 \mu \mathrm{m}$ in (c) and (d), respectively. (a) and (c) are measured using Fig. 1(a) configuration, whereas (b) and (d) are measured according to Fig. 1(b). The lattice constant of the hole array $a=9 \mu \mathrm{m}$.

$(-1,0) \mathrm{Ag} / \mathrm{Si}$ modes shown in Fig. 3(c) and the $(0, \pm 1)$ $\mathrm{Ag} / \mathrm{Si}$ mode in Fig. 3(d) disappear, but both the $(-1, \pm 1)$ $\mathrm{Ag} / \mathrm{Si}$ and the $(1, \pm 1) \mathrm{Ag} / \mathrm{Si}$ modes existed. Figure 4 shows the transmission spectrum of a square array of rectangular holes at normal incident with polarized light. When the aspect ratio of the rectangular hole was 1.15 , light polarized in the $y$ direction results in double peaks at 31.4 and $32.6 \mu \mathrm{m}$ because the width of rectangular holes, $4.7 \mu \mathrm{m}$, is close to half of the lattice constant, $4.5 \mu \mathrm{m}$. These double peaks are due to Bragg scattering of the SPPs on periodic perforated squared hole arrays which leads to two standing waves $\left(\omega_{+}\right.$ and $\left.\omega_{-}\right)$, each with its own surface charge energy, ${ }^{19,20}$ and splits into two peaks. The light polarized in the $x$ direction results in one peak at $31 \mu \mathrm{m}$ due to less Bragg scattering because the length of the rectangular holes, $5.4 \mu \mathrm{m}$, is away from the half of the lattice constant. ${ }^{21}$ Only two instead of three peaks are observed because the high energy peaks

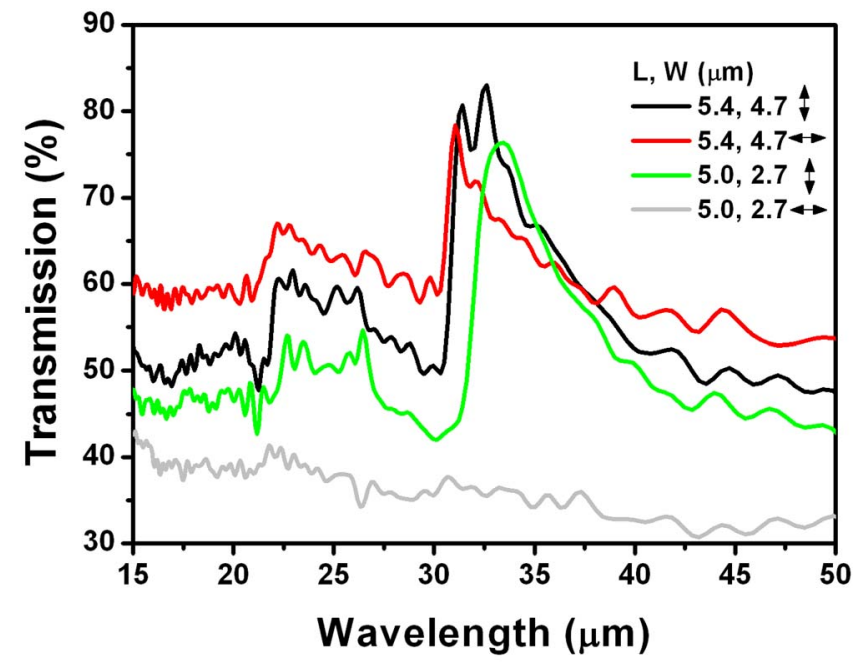

FIG. 4. (Color online) Zero-order transmission spectra of a squared hole array at normal incidence with polarized light. The lattice constant of the hole array $a=9 \mu \mathrm{m}$.

to AIP license or copyright; see http://apl.aip.org/apl/copyright.jsp 


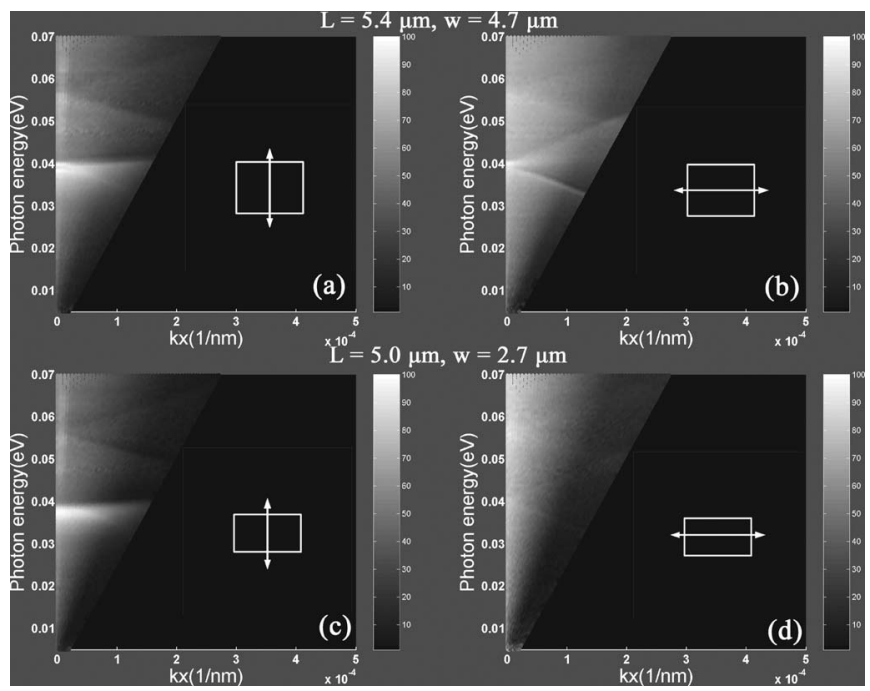

FIG. 5. Energy dispersion relation of SPPs, transmission intensity (gray scale) as a function of photon energy, and $\mathbf{k}_{x}$. The length and width of the rectangular hole are $L=5.4 \mu \mathrm{m}$ and $W=4.7 \mu \mathrm{m}$ in (a) and (b), and $L$ $=5.0 \mu \mathrm{m}$ and $W=2.7 \mu \mathrm{m}$ in (c) and (d), respectively. (a) and (c) are measured for light polarized perpendicular to the long edge, whereas (b) and (d) are measured for light polarized perpendicular to the short edge. All measurements are using Fig. 1(a) configuration. The lattice constant of the hole array $a=9 \mu \mathrm{m}$.

formed by two polarized lights are too close, that is, indistinguishable. When the aspect ratio of the rectangular hole was 1.9 , light polarized in the $y$ direction results in a peak at $33.5 \mu \mathrm{m}$ which slightly redshifts due to the coupling of LSP wave. But for light polarized in the $x$ direction, no SP mode is observed which implies that the SP modes are hardly induced in a direction perpendicular to short edges. The angular variation of the polarized light measured using Fig. 1(a) scheme is shown in Figs. 5(a)-5(d). When the aspect ratio of the rectangular hole was 1.15 and the polarized light is in the $y$ direction which is perpendicular to the long edge of the rectangular holes, this tends to induce charge dipole oscillation between the long edges ( $y$ axis) but not the short edges ( $x$ axis). As a result, the $(1,0) \mathrm{Ag} / \mathrm{Si}$ and $(-1,0) \mathrm{Ag} / \mathrm{Si}$ modes along the $x$ axis disappear, but the $(0, \pm 1) \mathrm{Ag} / \mathrm{Si}$ modes along the $y$ axis are induced, as shown in Fig. 5(a). When the electric field polarization is parallel to the long edges (in the $x$ direction), the double peaks are induced which come from the $(1,0) \mathrm{Ag} / \mathrm{Si}$ and $(-1,0) \mathrm{Ag} / \mathrm{Si}$ modes, as shown in Fig. 5(b). As mentioned above, the double peaks also imply the Bragg scattering of the SPPs on periodic perforated squared hole arrays which results in two standing waves $\left(\omega_{+}\right.$and $\left.\omega_{-}\right)$. When the aspect ratio of the rectangular hole was 1.9 , the light polarized in the $y$ direction which is perpendicular to long edge, the charge dipole oscillation between long edges of the rectangular hole along the $y$ axis is induced, i.e., the $(0, \pm 1) \mathrm{Ag} / \mathrm{Si}$ modes, as shown in Fig. 5(c), and the $(1,0) \mathrm{Ag} / \mathrm{Si}$ and $(-1,0) \mathrm{Ag} / \mathrm{Si}$ modes along the $x$ axis disappear. Figure 5(d) shows that when the polarized light is perpendicular to the short edge (in the $x$ direction), it is hard to induce charge dipole oscillation along the $x$ axis, no SP mode was observed. In Fig. 1(a), no matter what the light propagates along the $x$ or $y$ direction, the projection of the electric field in the $x-y$ plane is random. The $(-1, \pm 1)$ $\mathrm{Ag} / \mathrm{Si}$ and $(1, \pm 1) \mathrm{Ag} / \mathrm{Si}$ modes in the $x$ or $y$ direction are symmetric and the coupling efficiencies are the same. Therefore, both $(-1, \pm 1) \mathrm{Ag} / \mathrm{Si}$ and $(1, \pm 1) \mathrm{Ag} / \mathrm{Si}$ modes all exist. The charge dipole occurred between either the long or short rectangular hole edges can be distinguished by the light polarization, as shown in Figs. 5(a) and 5(b). If the aspect ratio of the rectangular hole is large, then the surface plasmon tends to couple strongly with the local charge dipole oscillations between the long edges even though the polarized light act on the short edges, it is hard to induced the dipole charge between short edges.

In conclusion, the SP dispersion relations of a square array of rectangular $\mathrm{Ag}$ holes with different aspect ratios are investigated. The aspect ratio of the rectangular hole increases, and strong coupling between LSPs and the long edges of each rectangular hole changes the cutoff wavelength and the transmission intensity, resulting in the disappearance of certain $\mathrm{Ag} / \mathrm{Si}$ modes when light propagates along short or long edges of the rectangular holes. The direction of the electric field oscillation and that of the light propagation both affected the SPP dispersion relation.

This work is supported by the National Science Council of Republic of China under Contact No. NSC 94-2215-E002-042.

${ }^{1}$ H. F. Ghaemi, Tineke Thio, D. E. Grupp, T. W. Ebbesen, and H. J. Lezec, Phys. Rev. B 58, 6779 (1998).

${ }^{2}$ T. W. Ebbesen, H. J. Lezec, H. F. Ghaemi, T. Thio, and P. A. Wolff, Nature (London) 391, 667 (1998).

${ }^{3}$ Shaun M. Williams, Amanda D. Stafford, Trisha M. Rogers, Sarah R. Bishop, and James V. Coe, Appl. Phys. Lett. 85, 1472 (2004).

${ }^{4}$ D. Egorov, B. S. Dennis, G. Blumberg, and M. I. Haftel, Phys. Rev. B 70, 033404 (2004).

${ }^{5}$ Shaum M. Williams, Amanda D. Stafford, Kerneth R. Rodriguez, Trisha M. Rogers, and James V. Coe, J. Phys. Chem. B 107, 11871 (2003).

${ }^{6}$ Y.-H. Ye and Jia-Yu Zhang, Appl. Phys. Lett. 84, 2977 (2004).

${ }^{7}$ Yong-Hong Ye and Jia-Yu Zhang, Opt. Lett. 30, 1521 (2005).

${ }^{8}$ A. Degiron and T. W. Ebbesen, J. Opt. A, Pure Appl. Opt. 7, S90 (2005).

${ }^{9}$ A. Degiron, H. J. Lezec, N. Yamamoto, and T. W. Ebbesen, Opt. Commun. 239, 61 (2004).

${ }^{10}$ K. L. van der Molen, K. J. Klein Koerkamp, S. Enoch, F. B. Segerink, N. F. van Hulst, and L. Kuipers, Phys. Rev. B 72, 045421 (2005).

${ }^{11}$ K. J. Klein Koerkamp, S. Enoch, F. B. Segerink, N. F. van Hulst, and L. Kuipers, Phys. Rev. Lett. 92, 183901 (2004).

${ }^{12}$ R. Gordon, A. G. Brolo, A. McKinnon, A. Rajora, B. Leathem, and K. L. Kavanagh, Phys. Rev. Lett. 92, 037401 (2004).

${ }^{13}$ Hua Cao and Ajay Nahata, Opt. Express 12, 3664 (2004).

${ }^{14}$ F. J. García-Vidal, E. Moreno, J. A. Porto, and L. Martín-Moreno, Phys. Rev. Lett. 95, 103901 (2005).

${ }^{15}$ R. Gordon and A. G. Brolo, Opt. Express 13, 1933 (2005).

${ }^{16}$ M. U. Pralle, N. Moelders, M. P. McNeal, I. Puscasu, A. C. Greenwald, J. T. Daly, E. A. Johnson, T. George, D. S. Choi, I. El-Kady, and R. Biswas, Appl. Phys. Lett. 81, 4685 (2002).

${ }^{17}$ Irina Puscasu, M. Pralle, M. McNeal, J. Daly, A. Greenwald, E. Johnson, R. Biswas, and C. G. Ding, J. Appl. Phys. 98, 013531 (2005).

${ }^{18}$ H. J. Lezec, A. Degiron, E. Devaux, R. A. Linke, L. Martin-Moreno, F. J. Garcia-Vidal, and T. W. Ebbesen, Science 297, 820 (2002).

${ }^{19}$ W. L. Barnes, T. W. Preist, S. C. Kitson, and J. R. Sambles, Phys. Rev. B 54, 6227 (1996).

${ }^{20}$ S. C. Kitson, W. L. Barnes, and J. R. Sambles, Phys. Rev. B 52, 11441 (1995).

${ }^{21}$ Ming-Wei Tsai, Tzu-Hung Chuang, Hsu-Yu Chang, and Si-Chen Lee, Appl. Phys. Lett. 88, 213112 (2006). 